\title{
ON THE USE OF FRACTAL CONCEPTS IN ANALYSIS OF DISTRIBUTIONS OF GALAXIES
}

\author{
I.B.VAVILOVA \\ Astronomical Observatory of Kiev University \\ Observatornaya str. 3 \\ Kiev 254053 Ukraine \\ e-mail:vil\%rosa.kiev.ua@relay.ua.net
}

The well-grounded polemics about the fractal structure of the Universe and a new cosmological picture which appears in connection with this, in first instance the absence of any evidence for homogenization up to present observational limits $200 \mathrm{~h}^{-1} \mathrm{Mpc}$, have been detailed at the work by Coleman, Pietronero (1992). Two versions on nature of fractal pattern of the galaxy distribution in the observed universe also are now: it behaves like a simple homogeneous fractal (Pietronero 1987; Coleman et al. 1988) and as a multifractal - fractal having more than one scaling index (Jones et al. 1988; Martinez, Jones 1990; Martinez et al. 1990; Borgani et al. 1993 (with the good review for matter of above)).

This work does not play decisively into hands of these versions so the fractal concepts, exactly a selfsimilarity and multifractal, were applied here for the analysis of two - dimensional distribution of the bright galaxies and dwarf galaxies of the low surface brightness (LSBD) belonging to the Local Supercluster (LS). But if the observed universe holds the fractal structure, so it is useful to trace over the lower fractal pattern on the small scales of clustering of galaxies within the framework of the known superclusters and, in the first instance, within the local overdensity of galaxies. This work is a preliminary before preparing one with the same analysis of three- dimensional distribution of galaxies of LS.

We included to consideration the bright and LSBD galaxies compiled from the Catalogue of LSBD galaxies (Karachentseva, Sharina, 1988), VCC (Binggeli et al. 1987) and FCC (Ferguson, Sandage 1990,1991) with the $V_{h} \leq 2000 \mathrm{~km} / \mathrm{s}$ : bright and LSBD's of the Virgo, $\mathrm{N}=974$; bright and LSBD's of the Fornax, $N=371$, LSBD's of all the Local Supercluster, $N=1714$. Concerning principles of selection and results on the clustering for these samples we refer to works by Karachentseva, Vavilova (1994a,b). We also generated a point distribution of galaxies according to the law of "Koch-curve" (pure fractal) to have a handle for the comparison with our results. The spherical coordinates of galaxies of all samples were transformed by using an equigraphic Mol'veide projection that is topologically invariant for this task.

For calculation of measure of the homogeneous fractal we chose a box-counting method providing a good accuracy for a finite point set. It is known that the fractal holds a straight--line behaviour at the plot of $\log N(\varepsilon)$ vs. $(\log 1 / \varepsilon)$ for the certain meanings of size $\varepsilon$ of box, here $N(\varepsilon)$ is the number of occupied boxes which are received by partitioning of sample. The slope for this part of curve is the fractal dimension D. Samples of galaxies of the Virgo and the Fornax clusters demonstrate scaling - dependent behaviour from the size $\varepsilon$ but have a little plateau (Fig. 1) which is a characteristic for the homogeneous fractal ( $D \sim 1.4$ and 1.15 accordingly). The distribution of LSBD's of all the LS appears a scale - independence at the scales $\varepsilon \sim 0.8^{\circ} \div 10^{\circ}$ when a statistical weight of group or cluster of galaxies coincides to the weight of field galaxy. 
Second fractal concept has been considered is the multifractal (we applied the work by Feder 1988). Analogously dividing in the box-counting method we calculated the number of galaxies $\mathrm{N}_{\mathrm{i}}(\varepsilon)$ within each cell and assigned a probability measure $\mathrm{P}_{\mathrm{i}}(\varepsilon)$ to occupy the $\mathrm{i}$ - cell. Neglecting the known formulas for breviary, note that moments of this measure define the function $\tau(q)$ which is related to the generalized dimension $D_{q}=\tau(q) /(q-1)$, where $D_{0}$ is the Hausdorff dimension, $\mathrm{D}_{1}$ - information dimension and $\mathrm{D}_{2}$ - correlation dimension. $\mathrm{D}_{\mathrm{q}}$ is always decreasing function and large values of $q$ coincide largest values of $P_{i}(\varepsilon)$, by other words the denser regions of the galaxy distribution. We also calculated the $f(\alpha)$ - spectrum which is connected with the variables $\tau(q)$ and $q$ through the Legendre transformation likewise to Gibbs formalism of thermodynamics. The curve $f(\alpha)$ has an unique maximum and value of the $f(\alpha)$ spectrum in that point is the fractal dimension $\mathrm{f}(\alpha(\mathrm{q}=0))=\mathrm{D}_{0}$. Also $\alpha_{\min }$ is the scaling exponent for the region of max concentration of galaxies and, opposite, $\alpha_{\max }$ is the scaling index of the poorer region. In the figure 2 we present the dependencies $D(q)$ vs. $q$ and $f(\alpha)$ vs. $\alpha$ for considered samples of galaxies on some length scale. All samples of galaxies demonstrate a weak dependence $D(q)$ from $q$. The similarity of the curves indicates on similarity of clustering properties of galaxies of the LS and maximum of the $f(\alpha)$ curves corresponds to $D_{0} \approx 1.2$ for the law of similarity with the $\gamma \approx 1.8$ (power law exponent of $\mathrm{N}$-order correlation functions). It would be an inappropriate to suggest an existence of any fractality for these samples at this stage without decision of question for completeness of these samples and working of three-dimensional analysis

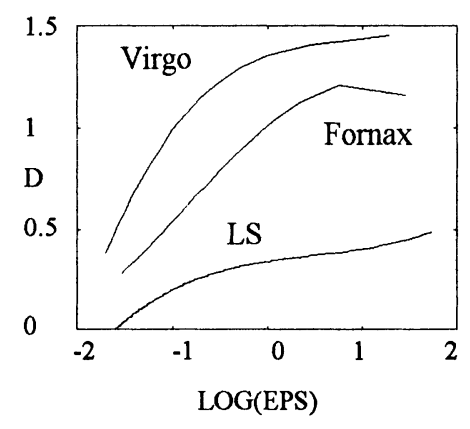

Fig.1.The scaling behaviour of fractal dimension $D$ with the size $\varepsilon$ for samples of galaxies of the Virgo and Fornax clusters and LSBD's of Local Supercluster. These graphes are received after the numerical differentiation and smoothing of curves $\log N(\varepsilon)$ vs. $\log (1 / \varepsilon)$.
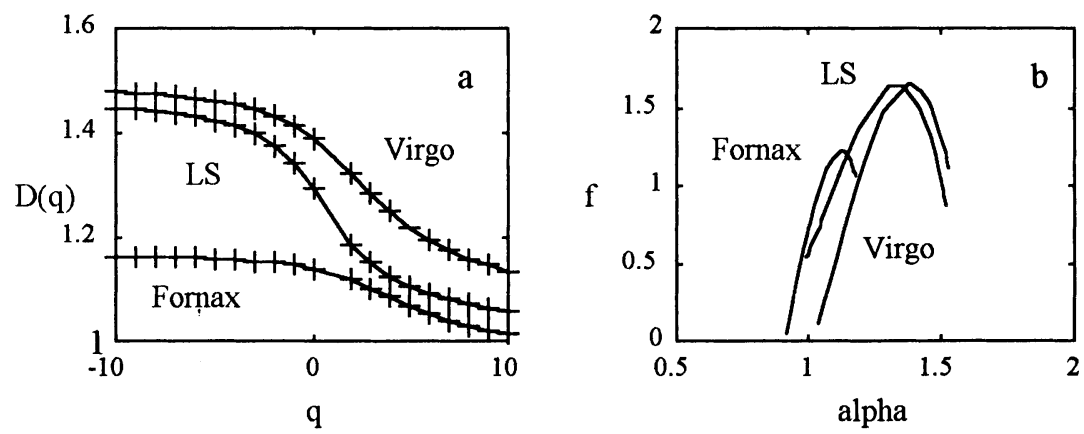

Fig.2. a) Generalized dimensions $D(q)$ and b) the $f(\alpha)$ - spectrum for the same samples. 


\section{Acknowledgment}

The author thanks Dr.V.E.Karachentseva, our joint working stimulated this work.

\section{References}

Binggeli, B., Tammann, G.A. \& Sandage, A., 1987. Astron.J., 94, 251.

Borgani, S., Plionis, M., \& Valdarnini, R., 1993. Astrophys. J., 404, 21

Coleman, P.H., Pietronero, L., 1992. Physics Rep., 213, 313.

Coleman, P.H., Pietronero, L. \& Sanders, R.H., 1988. Astron. \& Astrophys., 200, L32.

Feder J., 1988. Fractals (Plenum Press, New York).

Ferguson, H.C., Sandage, A., 1990. Astron.J., 100, 1.

Ferguson, H.C., Sandage, A., 1991. Astron.J, 101, 765.

Jones, B.J.T., Martinez, V.J., Saar, E., Einasto, J., 1988. Astrophys. J., 332, L1.

Karachentseva, V.E., Sharina, M.E., 1988. Commun. of SAO, 88, 1.

Karachentseva, V.E., Vavilova, I.B., 1994a. Bulletin of SAO, 37, 98.

Karachentseva, V.E., Vavilova, I.B., 1994b. In "Dwarf galaxies", ESO/OHP Workshop, France, 6-9 Sept., 1993, eds. J.Meylan and P.Prujniel, ESO Conf. \& Workshop Proc., No. 49, p.91.

Martinez, V.J. \& Jones, B.J.T., 1990. Mon. Not. R. astron. Soc., 242, 517.

Martinez, V.J., Jones, B.J.T., Domingues-Tenreiro, R., van de Weygaert, R., 1990. Astrophys. J., 357, 50.

Pietronero, L., 1987. Physica A, 144, 257. 